\title{
Freedom of medical prescription
}

\author{
Alberto Lifshitz, Oscar Arrieta, Carlos Campillo, Rubén Burgos, Miguel Ángel Celis, Manuel De la Llata, \\ Judith Domínguez, José Halabe, Sergio Islas, Luis Jasso, Mucio Moreno, Ricardo Plancarte, \\ Alejandro Reyes-Sánchez, Guillermo Ruiz-Argüelles, Antonio Soda, Emma Verástegui and Julio Sotelo* \\ Comité de Ética y Transparencia en la Relación Médico-Industria (CETREMI), Academia Nacional de Medicina de México, Ciudad de México, \\ Mexico
}

\begin{abstract}
Doctors require flexibility for prescription. However, some limits are laid down both, by current knowledge and by restrictions imposed by access, rules and regulations. The Committee for Ethics and Transparency in the Physician-Industry Relationship (CETREMI) of the National Academy of Medicine proposes several suggestions to help patients, which include the selection of the best alternatives for each case, formalization of prescription standards variations (doses, drug indications, etc.) written down in the medical records, and avoidance of fashions, untested novelties, argumentations solely based on advertising or commercial promotion and conflicts of interest.
\end{abstract}

KEY WORDS: Drugs. Prescription. Therapeutics. Drug promotion. Essential medicines list.

Doctors are very protective of their freedom to make decisions, assuming that the particular needs of each case are unique and only they have the elements to choose the best option. They resist complying with regulations that might limit this freedom. As long as the defense of this prerogative has to do with the well-being of their patients, under the principles of beneficence and autonomy, prescriptive freedom is legitimate, not so when it depends on defending an arrogant hierarchical position or when it answers to interests other than offering the patient the best alternative.

The boundaries of this freedom are naturally defined: by pharmacological knowledge, patient peculiarities, scientific evidence, physical existence of drugs and therapeutic technologies and access to them, which include protocols, standards and essential supplies lists. Access includes costs and is limited by prescription rules, which must be rational (selected by reason), reasoned (meditated, reflected on), reasonable (logical, appropriate), rationed (without waste) and rationalized (argumentatively justified).
The a priori concession to physicians of absolute trust in their technical and ethical authority has been confronted with the fact that they are human beings, with limitations deriving from the complexity of their profession, the influence of their own subjectivity, lack of expertise, limits of scientific knowledge and the possibility of making involuntary mistakes. This demystification is influenced by the fact that many professionals do not possess the technical and ethical qualities that the medical profession demands.

According to the opinion of the Committee of Ethics and Transparency in the Physician-Industry Relationship (CETREMI - Comité de Ética y Transparencia en la Relación Médico-Industria), a list of recommendations regarding the exercise of prescriptive freedom are presented:

1) Generic versus innovative drugs: the alternative has to do with costs, but also with the doctor's trust in certain products, derived from experience, evidence, commitments or effective promotion. It should be remembered that many generic drugs have undergone interchangeability tests
Correspondence:

*Julio Sotelo

E-mail: jsotelo@ unam.mx
Date of reception: 12-02-2019

Date of acceptance: 19-02-2019

DOI: 10.24875/GMM.M19000256
Gac Med Mex. 2019;155:186-187

Contents available at PubMed www.gacetamedicademexico.com 
and are called interchangeable generics (IGs) and can confidently substitute innovative drugs. The advantage of lower costs competes with the disadvantage of distrust. Many institutions have chosen to use interchangeable generics and the doctors who work for them are forced to use them. The pharmacy is not authorized to make substitutions that are not endorsed by doctors.

2) Modified treatment regimens: according to the circumstances and his/her own experience, the doctor can propose changes in standard treatment regimens, either in the dosage, administration route, fractionation, combinations or schedules. The issue is relevant, since many changes often lack bibliographic support and can lead to adverse effects. It is advisable for doctors to follow research-documented guidelines.

3) Non-validated or unauthorized usages: several drugs have properties additional to those they were authorized for; these properties are known as pleiotropic effects. Here again, for any non-validated or unauthorized use, the same suggestion is made as in previous paragraph: following a protocol, using research methodology and with reasonable justification expressed in the patient record.

4) Selection of commercial brands: the doctor has the freedom to choose the drug product. The criteria for this selection tend to be based on familiarity with the name, which usually derives from the publicity the manufacturer has made. If prescribing by active ingredient is chosen, the selection of a brand is exercised by the pharmacy clerk, which may be influenced by commercial incentives.

5) Veto of products: it has happened that, for commercial or interpersonal reasons, some colleagues have vetoed a certain commercial brand. Although this is within the freedom to choose, CETREMI has already ruled in this regard disqualifying this way of proceeding, especially when it comes to collegiate bodies. Regulatory agencies are the authorities that can issue alerts or withdraw products that are inconvenient for health from the market.

6) Adherence to standards, guidelines, algorithms and consensuses: except for certain mandatory standards, these documents are guidelines that help in decisions and it is the professionals' prerogative to comply with them or not. When not following them is decided, the recommendation is to explain the reasons in the patient record.

7) Essential medications lists: these are instruments for administrative control of health supplies. They are generally broad enough to cover most therapeutic needs, so it is preferable to make a selection from among those products therein listed; however, there should be flexibility to allow justified exceptions.

8) Interactions: foresight of interactions is an elementary condition that should guide prescription.

9) Costs: it is an ethical limitation to prescription freedom. Therapeutic decisions should be weighted taking the costs into account, in such a way that under equal circumstances the least expensive option is chosen.

10) Fashions and trends: it is common for advertising strategies to generate the fashion of using a certain medication. It should be borne in mind that not always new is best, or that the most expensive product is the best, or that the best advertised drug is the best, or that injected medications are better.

11) No medication: if a condition can be managed exclusively with other recommendations, concluding a medical consultation without prescribing a drug represents a favorable situation, since the risks associated with medication are avoided.

12) Routines, instinctive decisions, insistence of patients or relatives, pleasing patients or defensive practice: although these situations could be part of prescription freedom, the recommendation is avoiding them. 